\section{THE EFFECT OF DIFFERENT TYPES OF SPORTS ON RESTING HEART RATE VARIABILITY AND AUTONOMIC NERVOUS SYSTEM BALANCE}

\section{C.C. Grant and D.C. Janse van Rensburg}

\begin{abstract}
Heart rate variability (HRV) refers to the beat-to-beat variation in heart rate, largely due to fluctuating influences of the autonomic nervous system. There are indications that active participation in sport can alter the resting HRV and autonomic nervous system balance. The aim of this preliminary study was to determine whether different sports have different effects on autonomic balance, and whether HRV differs when top performers from their respective fields are compared. HRV measured in a control group and three groups of elite South African athletes were compared: endurance athletes, field hockey players and skydivers. Time domain, frequency domain and Poincaré analyses of baseline HRV were used to determine whether a difference exists between the three groups. Results were compared by means of a Kruskal-Wallis one way analysis of variance statistical test. This study indicated statistically significant differences between elite athletes from different sport types and a control group with regard to HRV and resting autonomic regulation of cardiac function. Of the three groups the endurance athletes had the highest HRV, as well as the highest vagal tone. The skydivers recorded the lowest HRV with sympathetic nervous system dominance. The control group and hockey players showed intermediate values between the other two groups for all parameters. Different types of sports influence resting autonomic balance to different extents. The results of this study point towards higher fitness levels, in terms of autonomic control of cardiac function in participants involved in aerobic-demanding sports activities. Lower HRV and increased sympathetic CV influence in the skydiving group may indicate increased dysrhythmogenic risk. Further studies conducted during non-participating periods and also in the later, postparticipation years are recommended.
\end{abstract}

Keywords: Autonomic nervous system, heart rate variability, elite athletes.

C. C. Grant (MSc Physiology)

D.C. Janse van Rensburg $(P h D)$
Section Sports Medicine

University of Pretoria

Pretoria 0002

South Africa

\section{E-mail: rina.grant@up.ac.za}

\section{INTRODUCTION}

Heart rate variability (HRV) refers to the beat-to-beat alterations in heart rate, largely due to fluctuating influences of the autonomic nervous system (ANS). The analysis of the HRV through linear and non-linear analytical techniques permits a non-invasive window on the ANS control (Aubert, Seps \& Beckers, 2003; Brown \& Brown, 2007). HRV seems to be a marker of both dynamic and cumulative load. HRV, as a dynamic marker of load, appears to be responsive and sensitive to acute mental and physical stress (Task Force, 1996; Brown, 2007). On the other hand, HRV as a marker of cumulative load reflects the wear and tear on the individual over longer periods and declines in ageing due to the decrease in efferent parasympathetic (vagal) tone and the reduced $\beta$-adrenergic responsiveness (Task Force, 1996; Gorman \& Slone, 2000). It is known that abnormal HRV may signal pathology. Decreased HRV as an indicator of abnormal autonomic control (reduced parasympathetic and/or increased sympathetic activity) of the heart 
is for instance known to go hand in hand with an increased risk for spontaneous ventricular arrhythmias (Gorman, 2000). Severely abnormal HRV, in general decreased HRV, is also associated with progression of coronary atherosclerosis, and deaths due to cardiac failure (Gorman, 2000). Decreased HRV has further been found in several other clinical conditions such as diabetic neuropathy, autonomic neuropathy, post heart transplantation, post myocardial infarction, and in certain psychiatric disorders (Gorman, 2000).

Certain factors and activities seem to increase HRV and therefore to benefit to the individual. Regular physical activity, such as endurance training has, for instance, been shown to increase HRV - presumably by increasing the vagal relative to the sympathetic influence on the heart (Eckblom, Küborn \& Soltysiak, 1973). It has, in fact, been shown that good aerobic fitness obtained by practicing sports could affect HRV by an absolute increase in parasympathetic power to the heart and one would presume that it may thus exert cardioprotective effects and in so-doing benefit the cardiovascular risk profile (Eckblom, 1973; Aubert, Beckers \& Ramaekers, 2001; Aubert, 2003). There are even indications that aerobic exercise training, by its ability to correct the autonomic balance, may be a useful adjuvant or an alternative to drug therapy in some cardiovascular diseases (Aubert, 2003). On the other hand, it is also known that when the body is stressed repetitively with insufficient time to recover, a condition known as overtraining may result (Karvonen, 1992). In overtraining the autonomic balance can show an excessive shift in the balance, either in the direction of parasympathetic or sympathetic (Aubert, 2003). The relationship between performance and training load, in general, is non-linear (Karvonen, 1992). This phenomenon probably also applies to the autonomic nervous system balance. In short, the potential benefit of training and fitness on the autonomic nervous system, and by implication on cardiovascular health, appears to be biphasic and it is reasonable to expect that the type of training would play a role in the influence on the autonomic nervous system. 
The aim of this study was to compare the autonomic nervous system functioning, as measured by $\mathrm{HRV}$, in three groups of elite South African athletes from totally different sports types and a control group. The question was asked whether a difference in resting autonomic nervous system balance really exists when comparing groups of athletes who are amongst the top performers in their respective sports and how does it compare with a control group of the same average age and weight. The three groups of athletes investigated in this study were field hockey players, endurance athletes (distance runners) and skydivers. Although all the participants were elite athletes, the sports in which they excel vary in the physical and mental demands required to reach the top. The demands for excellence in field hockey include aerobic endurance, muscular strength, good balance, flexibility, good hand-eye-coordination and superfast movement ability. Endurance-sport events such as distance running are known to require high aerobic power and the ability to maintain at a relatively high velocity over a long distance. The physical and mental abilities to excel in skydiving as a sport are often underestimated by other athletes as well as the general public. Depending on the level of competition, all of the above physical abilities play a role and must be performed under extreme stress.

\section{MATERIALS AND METHODS}

Participants

The study comprised ten South African Olympic field hockey players, ten skydiving athletes chosen to represent South Africa in a World Skydiving Meeting and 10 of the top South African Comrades athletes. The control group consisted of ten moderately active students from the University of Pretoria. Moderately active was defined as performing no more than 30 minute exercise sessions 2-3 days per week. All participants were free of any obvious cardiovascular abnormalities and none were on cardio-active medicines. They were all volunteers and provided written informed consent after the protocol was explained. Ethical clearance for the study was obtained. The physical characteristics of the participants are presented in Table 1. 
able 1: Physical characteristics of participants.

\begin{tabular}{|l|l|l|l|}
\hline Group & $\begin{array}{l}\text { Age } \\
(\mathbf{y r})\end{array}$ & $\begin{array}{l}\text { Stature } \\
(\mathbf{m})\end{array}$ & $\begin{array}{l}\text { Body } \\
\text { mass (kg) }\end{array}$ \\
\hline $\begin{array}{l}\text { Endurance } \\
\text { Athletes }\end{array}$ & $\begin{array}{l}26.2 \\
\text { (SD 2.8) }\end{array}$ & $\begin{array}{l}1.71 \\
\text { (SD 0.06) }\end{array}$ & $\begin{array}{l}57.80 \\
\text { (SD 6.24) }\end{array}$ \\
\hline $\begin{array}{l}\text { Hockey } \\
\text { Athletes }\end{array}$ & $\begin{array}{l}24.5 \\
\text { (SD 3.1) }\end{array}$ & $\begin{array}{l}1.73 \\
(\text { SD 0.9) }\end{array}$ & $\begin{array}{l}58.20 \\
\text { (SD 7.10) }\end{array}$ \\
\hline $\begin{array}{l}\text { Skydiver } \\
\text { Athletes }\end{array}$ & $\begin{array}{l}28.9 \\
\text { (SD 4.2) }\end{array}$ & $\begin{array}{l}1.70 \\
(\text { SD .05) }\end{array}$ & $\begin{array}{l}61.46 \\
\text { (SD 7.32) }\end{array}$ \\
\hline $\begin{array}{l}\text { Control } \\
\text { group }\end{array}$ & $\begin{array}{l}25.7 \\
\text { (SD 5.3) }\end{array}$ & $\begin{array}{l}1.72 \\
(\text { SD.08) }\end{array}$ & $\begin{array}{l}65.21 \\
\text { (SD 6.20) }\end{array}$ \\
\hline
\end{tabular}

\section{Procedure}

The heart rate variability was determined by analysis of the RR interval data sets (tachograms), which were obtained by POLAR NV heart rate monitors. The data, RR-intervals, were sampled while the athletes were lying supine in a quiet environment at a room temperature of 22 ?C. Recordings were made over a period of $15 \mathrm{~min}$, but only the middle third (5 minutes) of the recordings were used in this study. The athletes were instructed not to drink any alcohol or caffeine or to smoke in the preceding 24 hours. They were allowed to eat a low protein breakfast (cereal with milk) on the morning of testing.

\section{Quantification of Heart Rate Variability}

The data ( $R R$ interval sets) were analysed using HRV Analysis Software 1.1 for windows developed by The Biomedical Signal Analysis Group, Department of Applied Physics,
University of Kuopio, Finland. Smoothness priors for trend and Model Eye programme settings were used for detrending with an Alpha value of 500 (Task Force, 1996; Tarvainen, Rantaaho \& Karjalainen, 2002). The auto regressive model order value was 16 and the interpolation rate was $4 \mathrm{~Hz}$. The techniques used for the evaluation of HRV from RR-interval data sets, were grouped into three categories: time domain, frequency domain and nonlinear analysis

\section{Time domain analysis}

Statistical methods were used to quantify time domain HRV indicators: Mean RR; RMSSD; and pNN50 (Task Force, 1969; Aubert, 2003). The abbreviations are explained in Table 2.

Table 2: Time domain indicators of HRV

\begin{tabular}{|l|l|}
\hline $\begin{array}{l}\text { Mean RR } \\
\text { (s) }\end{array}$ & $\begin{array}{l}\text { The mean of the intervals between } \\
\text { successive QRS complexes, indicator of } \\
\text { vagal (short term) and sympathetic (long } \\
\text { term) influence on HRV }\end{array}$ \\
\hline SDRR (s) & $\begin{array}{l}\text { Standard deviation of intervals between } \\
\text { successive QRS complexes, indicator of } \\
\text { vagal (short term) and sympathetic (long } \\
\text { term) influence on HRV }\end{array}$ \\
\hline $\begin{array}{l}\text { RMSSD } \\
\text { (ms) }\end{array}$ & $\begin{array}{l}\text { Root mean square of the standard deviation } \\
\text { between RR intervals, indicator of vagal } \\
\text { influence (short term) on HRV }\end{array}$ \\
\hline pNN50 (\%) & $\begin{array}{l}\text { The percentage of successive RR interval } \\
\text { differences larger than 50ms computed over } \\
\text { the entire recording, indicator of vagal } \\
\text { influence, (short term) on HRV }\end{array}$ \\
\hline
\end{tabular}


Spectral analysis

Spectral analysis of HRV produces a decomposition of total variation of the data series into its frequency components, which can be expressed in the form of a spectral density function that depicts spectral power as a function of frequency (Aubert et al., 2001). In this study the spectral components analysed by frequency domain analysis included high frequency (HF), $0.15-$ $0.40 \mathrm{~Hz}$, low frequency (LF), $0.04-$ $0.15 \mathrm{~Hz}$, and the LF/HF ratio (Task Force, 1996; Gorman 2000; Aubert, 2003). The $\mathrm{LF} / \mathrm{HF}$ ratio is used to assess the fractional distribution of power and is an indicator of the cardiac autonomic balance (Task Force, 1996; Aubert, 2003).

\section{Poincaré analysis (Non-linear analysis)}

The Poincare plot is a scatter gram in which each RR-interval of a tachogram is plotted as a function of the previous one. The two quantitative indicators that were determined were SD1 and SD2 (Mourot, Bouhaddi, Perrey, Cappelle, Henriet \& Wolf, 2004).

\section{Statistical Analysis}

The 4 groups were compared by means of a Kruskal-Wallis one way analysis of variance statistical test, with a confidence level of $95 \%$.

\section{RESULTS}

The aim of this study was to quantify the autonomic function of a control group, hockey players, skydivers and endurance athletes, thus determining whether any significant differences exist between the heart rate variability indicators among the 4 groups (Table 3). The comparison of the LF (sympathetic and parasympathetic regulation) and HF (parasympathetic regulation) power distribution in a control group, endurance athletes, field hockey players and skydivers are seen in Figure 1.

\section{DISCUSSION}

This study looked at heart rate variability and distribution of autonomic power in a control group and three groups of elite sport performers, i.e., endurance athletes (long distance runners), hockey players and skydivers. It is generally assumed that aerobic fitness can favourably influence the autonomic control of the heart, but the question was asked whether differences can be seen in the distribution of the autonomic power when athletes from 


\section{GRANT AND JANSE VAN RENSBURG}

Table 3: Comparison between the time domain, frequency domain and non-linear HRV analysis of the control group $(C)$, sky-divers $(S)$, field hockey players $(H)$, endurance athletes $(A)$ and control group.

\begin{tabular}{|c|c|c|c|c|c|}
\hline $\begin{array}{c}\text { Analysi } \\
\text { S }\end{array}$ & Parameter & Group & Mean & p-value & Comment * \\
\hline \multirow{4}{*}{ 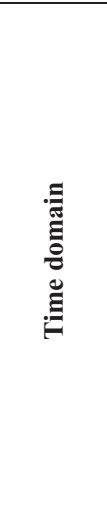 } & Mean RR & $\begin{array}{l}S \\
H \\
A \\
C\end{array}$ & $\begin{array}{l}0.84 \\
0.86 \\
0.99 \\
0.89 \\
\end{array}$ & 0.0373 & $\begin{array}{l}\text { S is significantly lower } \\
\text { than } A\end{array}$ \\
\hline & SDRR & $\begin{array}{l}S \\
H \\
A \\
C\end{array}$ & $\begin{array}{l}0.04 \\
0.05 \\
0.06 \\
0.04\end{array}$ & 0.0587 & No significant difference. \\
\hline & RMSSD & $\begin{array}{l}S \\
H \\
A \\
C\end{array}$ & $\begin{array}{l}31.73 \\
56.90 \\
85.59 \\
49.50\end{array}$ & 0.0316 & $\begin{array}{l}S \text { is significantly lower } \\
\text { than } A \text {. }\end{array}$ \\
\hline & $p N N 50$ & $\begin{array}{l}S \\
H \\
A \\
C\end{array}$ & $\begin{array}{l}19.07 \\
26.32 \\
46.62 \\
37.27\end{array}$ & 0.0382 & $\begin{array}{l}S \text { is significantly lower } \\
\text { than } A .\end{array}$ \\
\hline \multirow{2}{*}{ 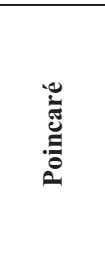 } & $\mathrm{SD} 1$ & $\begin{array}{l}S \\
H \\
A \\
C\end{array}$ & $\begin{array}{l}24.67 \\
40.47 \\
61.54 \\
35.90\end{array}$ & 0.0405 & $\begin{array}{l}S \text { is significantly lower } \\
\text { than } A .\end{array}$ \\
\hline & SD 2 & $\begin{array}{l}S \\
H \\
A \\
C\end{array}$ & $\begin{array}{l}68.62 \\
87.86 \\
97.81 \\
66.18\end{array}$ & 0.2325 & No significant difference. \\
\hline \multirow{3}{*}{ 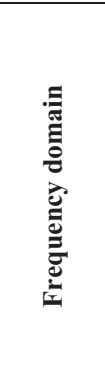 } & $\mathrm{LF}$ & $\begin{array}{l}S \\
H \\
A \\
C\end{array}$ & $\begin{array}{l}284.30 \\
479.80 \\
588.70 \\
293.60\end{array}$ & 0.2727 & No significant difference. \\
\hline & $\mathrm{HF}$ & $\begin{array}{l}S \\
H \\
A \\
C\end{array}$ & $\begin{array}{l}105.00 \\
248.80 \\
703.60 \\
223.50\end{array}$ & 0.0062 & $\begin{array}{l}S \text { is significantly lower } \\
\text { than } A .\end{array}$ \\
\hline & $\mathrm{LF} / \mathrm{HF}$ & $\begin{array}{l}S \\
H \\
A \\
C\end{array}$ & $\begin{array}{l}7.25 \\
1.81 \\
1.06 \\
1.88\end{array}$ & 0.0003 & $\begin{array}{l}S \text { is significantly higher } \\
\text { than } A, H \& C\end{array}$ \\
\hline
\end{tabular}

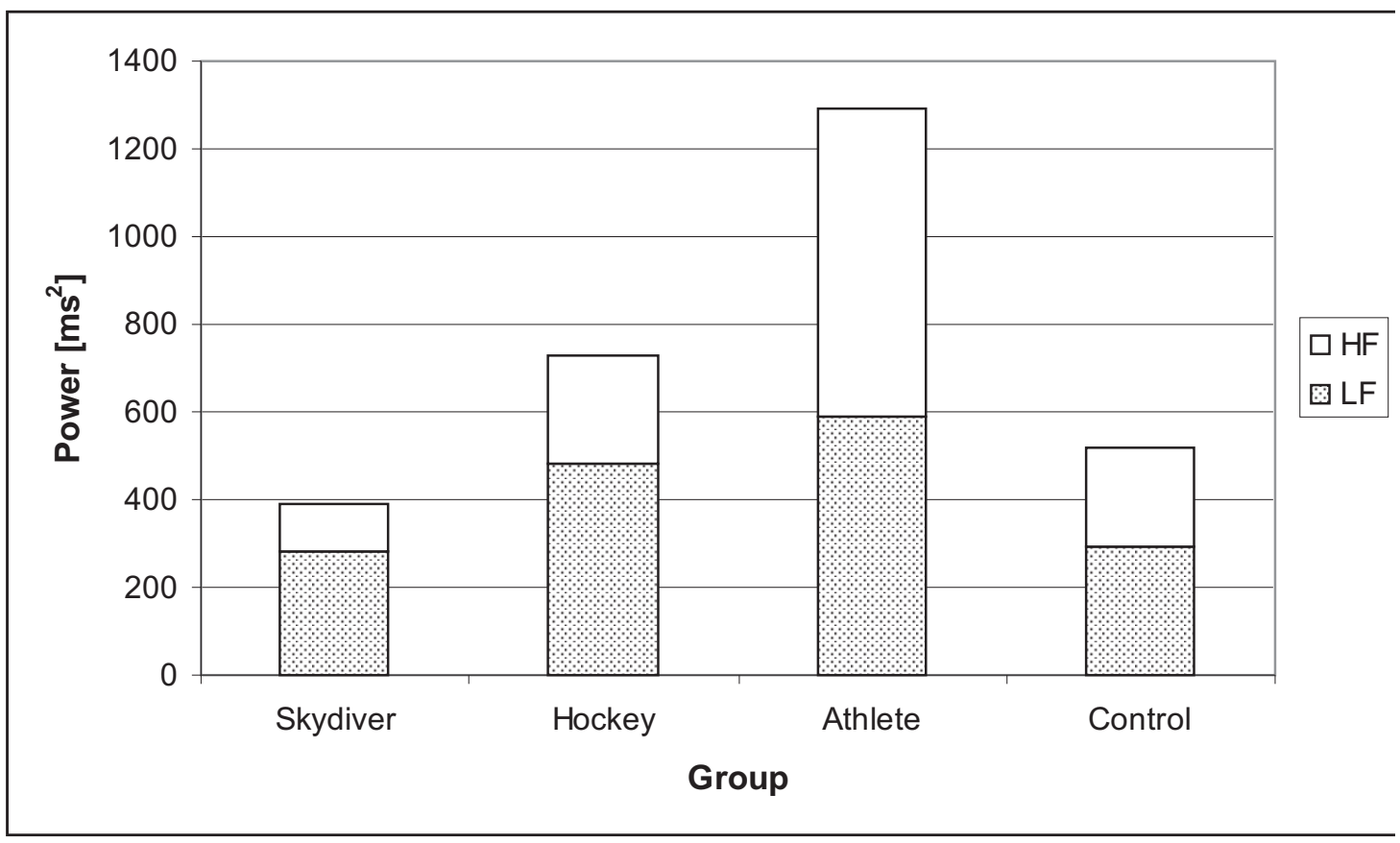

Figure 1: Comparison of the LF and HF power distribution in skydivers, field hockey players and endurance athletes 


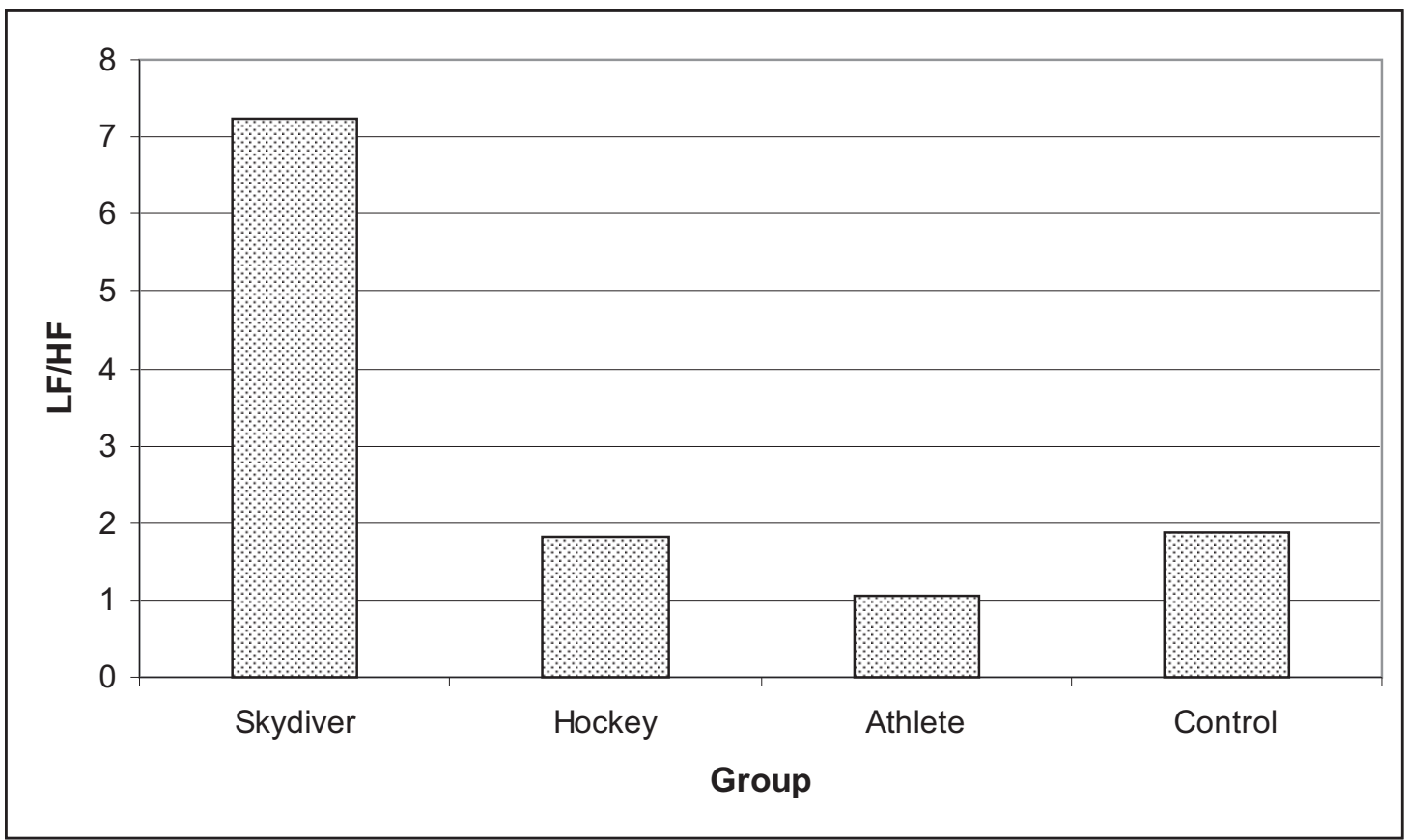

Figure 2: Comparison of the LF to HF ratio in skydivers, field hockey players and endurance athletes

HRV was analysed by time domain, frequency domain and Poincaré analysis. HRV indicators obtained by time domain analysis included firstly the mean RR and SDRR, both reflecting vagal, as well as sympathetic influences, and secondly the RMSSD and pNN50\%, both reflecting the vagal influence on HRV. In this study the mean RR $(p=0.0373), \quad R M S S D(p=0.0316)$ and pNN50 $\quad(\mathrm{p}=0.0382) \quad$ HRV indicators were significantly lower for the skydivers than for the control group, endurance athletes and hockey group. Time domain analysis thus reflected lower heart rate variability in skydivers than in the other three groups. It further indicated significant lower vagal influence in skydivers than in endurance athletes. HRV investigations by means of Poincaré analysis included the determination of SD1 and SD2 which reflect the short-term variations due to the parasympathetic, and the long termterm global variation, respectively (Mourot, 2004). Poincaré analysis supported the time domain indications of a lower vagal influence in skydivers than in endurance athletes. With respect to SD1 there was a significant difference $(p=0.0405)$ between the skydivers and the endurance athletes. HRV indicators obtained by frequency domain analysis included HF - known to primarily reflect the vagal influence, 
LF - which is determined by the combination of sympathetic as well as parasympathetic outflow to the heart, and the LF/HF ratio that reflects the balance or fractional distribution of power of the autonomic nervous system's influence on the heart (Task

Force, 1969; Aubert, 2003). No significant difference was found between the groups for the mean LF HRV indicator values. HF showed a significant difference $(p=0.0062)$ between the skydivers and the endurance athletes, supporting the results of the time domain and Poincaré analyses by pointing towards a significantly higher parasympathetic modulation of the heart in endurance athletes than in skydivers. When comparing the $\mathrm{LF} / \mathrm{HF}$ ratio which reflects the fractional distribution of power (sympathetic vs. parasympathetic) in this study, the skydivers' ratio was statistically significantly higher $(p=0.0003)$ than that of the control group, hockey players or the endurance athletes. This indicates significant increased sympathetic cardiovascular control in the skydivers relative to the rest (Figure 1).
This study showed that there are indeed differences between elite athletes from different sport types with regard to the resting autonomic regulation of cardiac function. The results of the endurance athletes support that of previous studies that showed high HRV and enhanced vagal tone in aerobically-fit athletes (Goldsmith, Bigger \& Steinmann, 1992; Dixon; Kamath \& McCartney, 1992; Aubert, Ramaekers \& Collier, 1999). Of the three groups, the endurance athletes had the highest HRV, as well as the highest vagal tone. The hockey players and control group showed intermediate values for all the analyses.

However, a reason for concern is the results of the third group, i.e., the skydivers. Results indicated significantly lower HRV with an increased LF/HF indicating sympathetic nervous system dominance and/or decreased vagal activity. It is known that autonomic imbalance plays an important role in progression of heart failure, and that increased LF/HF values $(\mathrm{LF} / \mathrm{HF}=5.3)$ is associated with congestive heart failure patients (Arai, 1989). 
The decreased HRV and altered autonomic balance indicated by an increase in $\mathrm{LF} / \mathrm{HF}$ ratio measured in the skydivers may point toward an increased cardiac dysrhythmic potential and an associated increased risk for cardiovascular incidents.

These results are not unexpected and several factors may have contributed. The main causes are probably the type of mental stress involved in the different sports, personality type and the differences in aerobic fitness - which is assumed to shift the autonomic power distribution towards parasympathetic. Skydiving is for instance often considered a sensation-seeking activity (Jack \& Ronan, 1998) and increased sympathetic activity during the freefall and even during preparation for skydiving (Chatterton, Vogelsong, Lu \& Hudgens, 1997) has been reported. Long-term aerobic physical training is said to influence cardiac rhythm, by inducing sinus bradycardia in resting conditions, as well as a shift of the sympathovagal balance towards parasympathetic dominance (Aubert et al., 2003). It is thus assumed that fitness, at least partially, underlies the enhanced vagal tone in endurance athletes and that it contributes to lower resting heart rates (Goldsmith et al., 1992). In general electrical stability of the heart is maintained through parasympathetic tone. Abnormal parasympathetic tone is directly linked to the occurrence of cardiac arrhythmias, and arrhythmias can occur when this tone is suddenly decreased (Verrier \& Dickerson, 1994). Normally, during stress situations, parasympathetic tone decreases, resulting in lowered HRV. However, sufficient parasympathetic influence remains to prevent the onset of arrhythmias (Verrier \& Dickerson, 1994).

It would thus appear that the higher the HRV and parasympathetic power, the more optimal the situation. However, as parasympathetic tone is associated with a cardiac deceleration and a lowering of blood pressure, high parasympathetic tone, or a strong shift towards parasympathetic dominance in the fractional distribution of autonomic power, is not necessarily always optimal and is implicated in several dysfunctional phenomena such as reduced perfusion, syncopy $(\mathrm{Li}, \mathrm{Hu} \&$ $\mathrm{Li}$, 1996) and perhaps symptoms of overtraining in athletes (Karvonen, 
1992; Aubert, 2003). It is wellrecognised that the majority of nontraumatic deaths in runners are caused by cardiovascular problems and a variety of causes are known to contribute (Sedivy, Bankl, Stimpfl, Bankl, \& Kurkciyan, 1997). There is also some evidence that syncopy may contribute to the exercise-associated collapse during endurance events with pooling of blood in the lower extremities described as the cause of the suggested syncopy (Holtzhausen, Noakes, Kroning, de Klerk, Roberts \& Emsley, 1994). In view of the role of the autonomic nervous system in syncopy (Li et al., 1996) and evidence that autonomic dysregulation may occur in overtraining (Aubert et al., 2003), it is feasible that such a dysregulation may also play a role in the exerciseassociated collapse of endurance events.

\section{CONCLUSIONS}

According to the results different types of sports have different effects on the resting autonomic regulation of cardiac function. This study showed the highest HRV values and a shift towards parasympathetic dominance in the endurance athletes and significantly lower HRV values and higher sympathetic sympathetic power in the skydivers. Results thus point towards higher HRV and a strong shift towards vagal dominance, in terms of resting autonomic control of cardiac function, in participants of the more aerobicdemanding sports activities. Here the question is what level of parasympathetic power is optimal for health as an excessive parasympathetic cardiac decelerating influence carries its own potential risk. As some of these results may suggest increased cardiovascular risk, longitudinal studies on these groups are suggested Perhaps it is necessary not only to further investigate the correlation between training, performance and autonomic balance during the periods of participation, but also the effects of the type of training on cardiac health during the non-participating periods and in the later, post-participation years.

\section{REFERENCES}

Arai,Y., Saul, J.P., Albrecht, P., Hartley, L,H., Lilly, L.S., Cohen, R.J. \& Collucci, W.S. Modulation of cardiac autonomic activity during and immediately after exercise. American Journal of Physiology, 256, 132-141.

Aubert, A.E., Beckers, F., Ramaekers, D. (2001). Short-term heart rate variability in young athletes. Journal of Cardiology, 37, Suppl. 1, 85-88.

Aubert, A.E., Ramaekers, D., Collier, B. \& Beckers, F. (1999). Comparison of the effect of different types of exercise on short-term HRV. Med Biol Eng Comput, 37, 568-569. 
Aubert, A.E., Seps, B. \& Beckers, F. (2003). Heart Rate Variability in Athletes. Sports Medicine, 33(12), 889-919.

Brown, S.J. \& Brown, J.A., (2007). Resting and post-exercise cardiac autonomic control in trained master athletes. Journal of Physiological Sciences, 57(1), 23-29.

Chatterton, R.T., Vogelsong, K.M., Lu, Y.C. \& Hudgens, G.A. (1997). Hormonal responses to psychological stress in men preparing for skydiving. Journal of Clinical Endocrinology and Metabolism, 82(8), 2503-2509.

Dixon, E.M., Kamath, M.V. \& McCartney, N. (1992). Neural regulation of HRV in endurance athletes and sedentary controls. Cardiovascular Research, 26(7), 713-719.

Ekblom, B., Küborn, A. \& Soltysiak, J. (1973). Physical training, bradycardia and autonomic nervous system. Scandinavian Journal of Clinical and Laboratory Investigation, 32, 251256.

Goldsmith, R.L.; Bigger Jr, J.T. \& Steinmann, R.C. (1992). Comparison of 24-hour parasympathetic activity in endurance-trained and untrained young men. Journal of the American College of Cardiology, 20(3), 552528.

Gorman, J.M. \& Sloan, R., P. (2000). Heart rate variability in depressive and anxiety disorders. American Heart Journal,140 (4 Suppl),77-83.

Holtzhausen, L.M., Noakes, T.D., Kroning, B., de Klerk, M., Roberts, M. \& Emsley, R. (1994). Clinical and biochemical characteristics of collapsed ultramarathon runners. Medicine and Science in Sports and Exercise. 26(9), 10951101.

Jack, S.J. \& Ronan, K.R. (1998). Sensation seeking among high- and low-risk sports participants. Personality and Individual Differences, 25(6), 1063-1083.

Karvonen, J. (1992). Overtraining. Med Sport Sci, 35, 174-188.

Li, S., Hu, D. \& Li, Y. (1996). Response and mechanism of vasovagal syncope induced by tilt table test. Chung - Hua Nei Ko Tsa Chih Chinese Journal of Internal Medicine, 35(3), $160-162$.
Mourot, L., Bouhaddi, M., Perrey, S., Cappelle, S., Henriet, M.T. \& Wolf, J.P. (2004). Decrease in heart rate variability with overtraining: Assessment by the Poincaré plot analysis. Clinical Physiology and Functional Imaging, 24(1), 10-18.

Sedivy, R., Bankl, H.C., Stimpfl, T., Bankl, H. \& Kurkciyan, I. Sudden (1997) unexpected death of a young marathon runner as a result of bronchial malformation. Modern Pathology, 10(3), 247-251.

Tarvainen, M. P., Ranta-aho, P.O. \& Karjalainen, P.A. (2002). An advanced detrending method with application to HRV analysis. IEEE Transactions on Biomedical Engineering, 49(2), 172 - 175.

Task Force of the European Society of Cardiology and the North American Society of Pacing and Electrophysiology. Heart Rate Variability, Standards of Measurement, Physiological Interpretation and Clinical use (1996). Circulation, 93, 1043-1065.

Verrier, R.L. \& Dickerson, L.W. (1994). Central nervous system and behavioral factors in vagal control of cardiac arrhythmogenesis. In M.M. Levy, P.J. Schwartz Vagal Control of the Heart (pp. 557-577). Armonk (NY): Futura. 